\title{
Capitulation des 2-classes d'idéaux de certains corps biquadratiques cycliques
}

\author{
par \\ Abdelmalek Azizi et Mohammed Talbi (Oujda)
}

1. Introduction. Soient $M$ un corps de nombres de degré fini sur $\mathbb{Q}$, $L$ une extension non ramifiée de $M$ et $p$ un nombre premier. L'extension $M^{(1)}$ de $M$, abélienne maximale et non ramifiée pour tous les idéaux premiers, finis et infinis, est dite le corps de classes de Hilbert de $M$. De même l'extension $M_{p}^{(1)}$ de $M$ dont le degré est une puissance de $p$, abélienne maximale et non ramifiée pour tous les idéaux premiers, finis et infinis, est dite le $p$-corps de classes de Hilbert de $M$.

La recherche des idéaux de $M$ qui capitulent dans $L$ (deviennent principaux dans $L$ ) a été l'objet d'étude de plusieurs mathématiciens. En effet, Kronecker était parmi les premiers à avoir abordé des problèmes de capitulation dans le cas des corps quadratiques imaginaires. Dans le cas où $L$ est égal au corps de classes de Hilbert $M^{(1)}$ de $M$, D. Hilbert avait conjecturé que toutes les classes de $M$ capitulent dans $M^{(1)}$ (théorème de l'idéal principal). La preuve de ce dernier théorème a été réduite par E. Artin à un problème de la théorie des groupes, et c'est Ph. Furtwängler qui l'avait achevée.

Le cas où $L / M$ est une extension cyclique et $[L: M]=p$, un nombre premier, a été traité par Hilbert. Sa réponse est le sujet du "théorème 94" qui affirme qu'il y a au moins une classe non triviale dans $M$ qui capitule dans $L$. De plus, Hilbert avait trouvé le résultat suivant : Soient $\sigma$ un générateur du groupe de Galois de $L / M, N_{L / M}$ la norme de $L / M, E_{M}$ le groupe des unités de $M, E_{L}$ celui de $L$ et $E_{L}^{*}$ le sous-groupe des unités de $E_{L}$ dont la norme, relative à l'extension $L / M$, est égale à 1 . Alors le groupe des classes de $M$ qui capitulent dans $L$ est isomorphe au groupe quotient $E_{L}^{*} / E_{L}^{1-\sigma}=H^{1}\left(E_{L}\right)$, le groupe cohomologique de dimension 1 .

À l'aide de ce théorème et de plusieurs résultats sur les groupes cohomologiques des unités, on obtient :

2000 Mathematics Subject Classification: 11R37, 11R29, 11R16.

A. Azizi est membre de l'Académie Hassan II des Sciences et Techniques, Maroc. 
ThÉORÈme 1 (voir [10]). Soit $L / M$ une extension cyclique non ramifiée de degré un nombre premier. Alors le nombre des classes qui capitulent dans $L / M$ est égal à

$$
[L: M]\left[E_{M}: N_{L / M}\left(E_{L}\right)\right]
$$

Aussi on note les travaux suivants sur des corps ayant une 2-partie du groupe de classes de type $(2,2)$. H. Kisilevsky [13] a étudié la capitulation pour les corps de nombres ayant un 2-groupe des classes de type $(2,2)$. En utilisant un calcul sur le transfert, il a lié le problème de capitulation à la structure du groupe de Galois de $M_{2}^{(2)} / M$. A. Azizi [1] a étudié la capitulation des corps $M=\mathbb{Q}(\sqrt{d}, i)$ où $i=\sqrt{-1}$ et $d$ est un entier naturel sans facteurs carrés et tel que le 2 -groupe de classes de $M$ est de type $(2,2)$; en utilisant les unités, il a trouvé le nombre des classes de $M$ qui capitulent dans les sous-extensions de $M_{2}^{(1)} / M$, ensuite il a déterminé la structure du groupe de Galois de $M_{2}^{(2)} / M$. Enfin A. Azizi et A. Mouhib $[4,5]$ ont étudié le même problème pour les corps $M=\mathbb{Q}(\sqrt{2}, \sqrt{d})$ où $d$ est un entier naturel sans facteurs carrés dont le 2-groupe de classes est de type $(2,2)$.

Soient $K=k(\sqrt{-p q \varepsilon \sqrt{2}})$ où $k=\mathbb{Q}(\sqrt{2})$, $\varepsilon$ l'unité fondamentale de $k$, $p$ et $q$ deux nombres premiers différents tels que $p \equiv q \equiv \pm 1 \bmod 4$ et $\left(\frac{2}{p}\right)=\left(\frac{2}{q}\right)=-1, K_{2}^{(1)}$ le 2 -corps de classes de Hilbert de $K, K_{2}^{(2)}$ le 2 -corps de classes de Hilbert de $K_{2}^{(1)}$ et $G$ le groupe de Galois de $K_{2}^{(2)} / K$. D'après E. Brown et C. J. Parry [7], la 2-partie $C_{2, K}$ du groupe de classes de $K$ est de type $(2,2)$, par suite $K_{2}^{(1)}$ contient trois extensions $F_{i} / K, i=1,2,3$.

Dans ce papier, on s'intéresse au problème de capitulation des 2-classes d'idéaux de $K$ dans $F_{i}(i=1,2,3)$ et à déterminer la structure de $G$. En particulier, on démontre le résultat suivant (théorèmes 7-9) :

ThÉORÈme 2 . Soient $K=k(\sqrt{-p q \varepsilon \sqrt{2}})$ où $\varepsilon$ est l'unité fondamentale de $k=\mathbb{Q}(\sqrt{2})$, p et $q$ deux nombres premiers différents tels que $p \equiv q \equiv$ $\pm 1 \bmod 4$ et $\left(\frac{2}{p}\right)=\left(\frac{2}{q}\right)=-1, K_{0}=\mathbb{Q}(\sqrt{2}, \sqrt{p q}), Q_{K_{0}}$ l'indice des unités de $K_{0}, F_{1}=K(\sqrt{p q}), F_{2}=K\left(\sqrt{p^{*}}\right), F_{3}=K\left(\sqrt{q^{*}}\right)$ avec $p^{*}=(-1)^{(p-1) / 2} p$, $q^{*}=(-1)^{(q-1) / 2} q$ et $G=\operatorname{Gal}\left(K_{2}^{(2)} / K\right)$. Alors :

(1) Si $p \equiv q \equiv-1 \bmod 4$, alors les quatre classes de $C_{2, K}$ capitulent dans chacune des extensions $F_{i}(i=1,2,3)$ et le groupe $G$ est abélien $(\simeq \mathbb{Z} / 2 \mathbb{Z} \times \mathbb{Z} / 2 \mathbb{Z})$.

(2) Si $p \equiv q \equiv 1 \bmod 4$, alors on $a$ :

(i) Si $Q_{K_{0}}=1$, alors les quatre classes de $C_{2, K}$ capitulent dans $F_{1}$, tandis que dans chaque $F_{i}, i \in\{2,3\}$, deux classes seulement de $C_{2, K}$ capitulent et le groupe $G$ est diédral d'ordre $2^{m}(m \geq 3)$. 
(ii) Si $Q_{K_{0}}=2$, alors dans chaque extension $F_{i}, i \in\{1,2,3\}$, il existe exactement deux classes de $C_{2, K}$ qui capitulent et le groupe $G$ est quaternionique d'ordre $2^{m}(m \geq 3)$.

\section{Préliminaires}

DÉfinition 1. On appelle corps de genres d'un corps de nombres $K$, qu'on note $K^{(*)}$, la plus grande extension de $K$ de la forme $K L$ qui est non ramifiée pour tous les idéaux premiers de $K$, finis et infinis, et telle que $L$ est une extension abélienne de $\mathbb{Q}$.

Proposition 1 (voir [11]). Soit $K$ un corps de nombres abélien sur $\mathbb{Q}$, de degré $n$. Si $n=q^{s}$ où $q$ est un nombre premier et $s>0$, alors

$$
K^{(*)}=\left(\prod_{p \mid D_{K}, p \neq q} L(p)\right) K
$$

où $L(p)$ est l'unique sous-corps, de degré e $(p)$ (l'indice de ramification de $p$ dans $K$ ) sur $\mathbb{Q}$, de $\mathbb{Q}\left(\xi_{p}\right)$ (le p-ième corps cyclotomique) et $D_{K}$ le discriminant de $K$.

Corollaire 1. Soient $K=k(\sqrt{-p q \varepsilon \sqrt{2}})$ où $k=\mathbb{Q}(\sqrt{2})$, $\varepsilon$ l'unité fondamentale de $k, p$ et $q$ deux nombres premiers impairs différents. Alors $K^{(*)}=K\left(\sqrt{p^{*}}, \sqrt{q^{*}}\right)$ où $p^{*}=(-1)^{(p-1) / 2} p$ et $q^{*}=(-1)^{(q-1) / 2} q$.

Démonstration. Comme les nombres premiers qui divisent $D_{K}$ sont $p, q$ et 2 , la proposition 1 montre que $K^{(*)}=L(p) L(q) K$, où $L(p)(\operatorname{resp} . L(q))$ est l'unique sous-corps, de degré 2 sur $\mathbb{Q}$, de $\mathbb{Q}\left(\xi_{p}\right)$ (resp. $\mathbb{Q}\left(\xi_{q}\right)$ ), et comme $L(p)=\mathbb{Q}\left(\sqrt{p^{*}}\right)\left(\right.$ resp. $\left.L(q)=\mathbb{Q}\left(\sqrt{q^{*}}\right)\right)$, on a le résultat.

Dans toute la suite de cette section on désigne par $K$ un corps de nombres, $C_{K}$ son groupe de classes, $C_{2, K}$ la 2-partie de $C_{K}, K_{2}^{(1)}$ le 2corps de classes de Hilbert de $K, K_{2}^{(2)}$ le 2-corps de classes de Hilbert de $K_{2}^{(1)}, G$ le groupe de Galois de $K_{2}^{(2)} / K$ et $G^{\prime}$ son sous-groupe dérivé. Alors $G^{\prime} \simeq \operatorname{Gal}\left(K_{2}^{(2)} / K_{2}^{(1)}\right)$ et $G / G^{\prime} \simeq \operatorname{Gal}\left(K_{2}^{(1)} / K\right)$, et on sait par la théorie des corps de classes que $\operatorname{Gal}\left(K_{2}^{(1)} / K\right) \simeq C_{2, K}$, ainsi $G / G^{\prime} \simeq C_{2, K}$.

Soient $F$ une extension cyclique non ramifiée de $K$ et $j$ l'application de $C_{K}$ dans $C_{F}$ qui fait correspondre à la classe d'un idéal $\mathcal{A}$ de $K$, la classe de l'idéal engendré par $\mathcal{A}$ dans $F$; et soit $N$ la norme de $F / K$. Alors, on dit que l'extension $F / K$ est :

- de type $(A)$ si $\left|\operatorname{ker} j \cap N\left(C_{F}\right)\right|>1$,

- de type $(B)$ si $\left|\operatorname{ker} j \cap N\left(C_{F}\right)\right|=1$.

DÉFInition 2. Soient $m$ un entier $>2, Q_{m}$ le groupe des quaternions, $D_{m}$ le groupe diédral et $S_{m}$ le groupe semi-diédral d'ordre $2^{m}$. Ces groupes 
sont définis comme suit. Chaque groupe est engendré par deux éléments $x$ et $y$ tels que :

$$
\begin{aligned}
Q_{m}=\langle x, y\rangle & \text { où } \quad x^{2^{m-2}}=y^{2}=a, \quad a^{2}=1, y^{-1} x y=x^{-1}, \\
D_{m}=\langle x, y\rangle & \text { où } \quad x^{2^{m-1}}=y^{2}=1, \quad y^{-1} x y=x^{-1} \\
S_{m}=\langle x, y\rangle & \text { où } \quad x^{2^{m-1}}=y^{2}=1, \quad y^{-1} x y=x^{2^{m-2}-1} .
\end{aligned}
$$

Supposons maintenant que $C_{2, K} \simeq \mathbb{Z} / 2 \mathbb{Z} \times \mathbb{Z} / 2 \mathbb{Z}$; alors $G / G^{\prime} \simeq \mathbb{Z} / 2 \mathbb{Z} \times$ $\mathbb{Z} / 2 \mathbb{Z}$, ce qui donne que $G^{\prime}$ est cyclique. Donc la tour des 2 -corps de classes de Hilbert de $K$ s'arrête en $K_{2}^{(2)}$.

En plus, on sait que si $G$ est d'ordre $2^{m}, m>1$, et $G / G^{\prime} \simeq \mathbb{Z} / 2 \mathbb{Z} \times \mathbb{Z} / 2 \mathbb{Z}$, alors, soit $G$ est isomorphe à $Q_{m}, D_{m}$, ou $S_{m}(m>2)$, soit à $\mathbb{Z} / 2 \mathbb{Z} \times \mathbb{Z} / 2 \mathbb{Z}$ $(m=2)$. Dans tous ces cas, on a $G^{\prime}=\left\langle x^{2}\right\rangle$ et les trois sous-groupes d'indice 2 dans $G$ sont : $H_{1}=\langle x\rangle, H_{2}=\left\langle x^{2}, y\right\rangle$ et $H_{3}=\left\langle x^{2}, x y\right\rangle$, et si $G^{\prime} \neq 1$, alors $K_{2}^{(1)} \neq K_{2}^{(2)}$ et $\left\langle x^{4}\right\rangle$ est l'unique sous-groupe de $G^{\prime}$ d'indice 2.

Soient $L$ le sous-corps de $K_{2}^{(2)}$ laissé fixe $\operatorname{par}\left\langle x^{4}\right\rangle, F_{i}(i=1,2,3)$ le souscorps de $K_{2}^{(2)}$ laissé fixe par $H_{i}$, et $j_{i}$ l'application $j$ définie pour $F=F_{i}$.

ThÉORÈme 3 . On suppose que $G / G^{\prime} \simeq \mathbb{Z} / 2 \mathbb{Z} \times \mathbb{Z} / 2 \mathbb{Z}$. Alors on $a$ :

(i) Si $K_{2}^{(1)}=K_{2}^{(2)}$, alors les corps $F_{i}$ sont de type $(A)$, $\mid$ ker $j_{i} \mid=4$ pour $i=1,2,3$ et $G \simeq \mathbb{Z} / 2 \mathbb{Z} \times \mathbb{Z} / 2 \mathbb{Z}$.

(ii) Si $\operatorname{Gal}(L / K) \simeq Q_{3}$, alors les corps $F_{i}$ sont de type $(A)$, $\left|\operatorname{ker} j_{i}\right|=2$ pour $i=1,2,3$ et $G \simeq Q_{3}$.

(iii) $S i \operatorname{Gal}(L / K) \simeq D_{3}$, alors les corps $F_{2}$ et $F_{3}$ sont de type $(B)$ et $\left|\operatorname{ker} j_{2}\right|=\left|\operatorname{ker} j_{3}\right|=2$. De plus, si $F_{1}$ est de type $(B)$ alors $\left|\operatorname{ker} j_{1}\right|=2$ et $G \simeq S_{m}$. Si $F_{1}$ est de type $(A)$ et $\left|\operatorname{ker} j_{1}\right|=2$, alors $G \simeq Q_{m}$. Enfin, si $F_{1}$ est de type $(A)$ et $\left|\operatorname{ker} j_{1}\right|=4$, alors $G \simeq D_{m}$.

On trouve plus d'information sur ce dernier théorème dans [13].

Corollaire 2. Soit $K$ tel que $C_{2, K} \simeq \mathbb{Z} / 2 \mathbb{Z} \times \mathbb{Z} / 2 \mathbb{Z}$. Alors on a trois types de capitulation:

- Type 1 : Les quatre classes de $C_{2, K}$ capitulent dans chacune des extensions $F_{i} / K, i=1,2,3$. Ceci est possible si et seulement si $K_{2}^{(2)}=K_{2}^{(1)}$.

- Type 2: Les quatre classes de $C_{2, K}$ capitulent toutes seulement dans une extension parmi les trois extensions $F_{i} / K, i=1,2,3$. Dans ce cas le groupe $G$ est diédral.

- Type 3: Seulement deux classes capitulent dans chacune des extensions $F_{i} / K, i=1,2,3$. Dans ce cas le groupe $G$ est semi-diédral ou quaternionique. 


\begin{tabular}{cccc}
\hline$\left|\operatorname{ker} j_{1}\right|(A / B)$ & $\left|\operatorname{ker} j_{2}\right|(A / B)$ & $\left|\operatorname{ker} j_{3}\right|(A / B)$ & $G$ \\
\hline 4 & 4 & 4 & $(2,2)$ \\
$2 A$ & $2 A$ & $2 A$ & $Q_{3}$ \\
4 & $2 B$ & $2 B$ & $D_{m}, m \geq 3$ \\
$2 A$ & $2 B$ & $2 B$ & $Q_{m}, m>3$ \\
$2 B$ & $2 B$ & $2 B$ & $S_{m}, m \geq 3$ \\
\hline
\end{tabular}

\section{Unités de certains corps de nombres de degré 4 ou 8 sur $\mathbb{Q}$.} Soient $d_{1}$ et $d_{2}$ deux entiers naturels sans facteurs carrés et premiers entre eux, $d_{3}=d_{1} d_{2}, \varepsilon_{1}$ (resp. $\left.\varepsilon_{2}, \varepsilon_{3}\right)$ l'unité fondamentale de $k_{1}=\mathbb{Q}\left(\sqrt{d_{1}}\right)$ (resp. $\left.k_{2}=\mathbb{Q}\left(\sqrt{d_{2}}\right), k_{3}=\mathbb{Q}\left(\sqrt{d_{3}}\right)\right), K_{0}=k_{1} k_{2}, Q_{K_{0}}$ l'indice des unités de $K_{0}$, et $N_{1}$ (resp. $N_{2}, N_{3}$ ) la norme de $K_{0} / k_{1}$ (resp. $K_{0} / k_{2}, K_{0} / k_{3}$ ).

On sait d'après [14] et [15] qu'un système fondamental d'unités (SFU) de $K_{0}$ est, à une permutation près des indices, l'un des systèmes suivants :

(i) $\left\{\varepsilon_{1}, \varepsilon_{2}, \varepsilon_{3}\right\}$;

(ii) $\left\{\varepsilon_{1}, \varepsilon_{2}, \sqrt{\varepsilon_{3}}\right\} \quad\left(N_{2}\left(\varepsilon_{3}\right)=1\right)$;

(iii) $\left\{\sqrt{\varepsilon_{1} \varepsilon_{2}}, \varepsilon_{2}, \varepsilon_{3}\right\}$

$\left(N_{3}\left(\varepsilon_{1}\right)=N_{3}\left(\varepsilon_{2}\right)=1\right) ;$

(iv) $\left\{\varepsilon_{1}, \sqrt{\varepsilon_{2}}, \sqrt{\varepsilon_{3}}\right\}$

$\left(N_{1}\left(\varepsilon_{2}\right)=N_{1}\left(\varepsilon_{3}\right)=1\right) ;$

(v) $\left\{\sqrt{\varepsilon_{1} \varepsilon_{2}}, \sqrt{\varepsilon_{2} \varepsilon_{3}}, \sqrt{\varepsilon_{1} \varepsilon_{3}}\right\}$

$\left(N_{2}\left(\varepsilon_{3}\right)=N_{3}\left(\varepsilon_{j}\right)=1, j=1,2\right)$;

(vi) $\left\{\sqrt{\varepsilon_{1} \varepsilon_{2} \varepsilon_{3}}, \varepsilon_{2}, \varepsilon_{3}\right\}$

$\left(N_{3}\left(\varepsilon_{1}\right)=N_{3}\left(\varepsilon_{2}\right)=N_{2}\left(\varepsilon_{3}\right)= \pm 1\right)$.

Proposition 2 (voir [2]). Soient $K_{0}$ un corps de nombres abélien réel et $\beta$ un entier algébrique de $K_{0}$, positif, sans facteurs carrés. On suppose que $K=K_{0}(\sqrt{-\beta})$ est une extension quadratique de $K_{0}$, abélienne sur $\mathbb{Q}$ et que $i=\sqrt{-1}$ n'appartient pas à $K$. Soit $\left\{\varepsilon_{1}, \ldots, \varepsilon_{r}\right\}$ un SFU de $K_{0}$. On choisit, sans restreindre la géneralité, les unités $\varepsilon_{j}$ positives. Alors on a :

(1) S'il existe une unité de $K_{0}$ de la forme $\varepsilon=\varepsilon_{1}^{j_{1}} \cdots \varepsilon_{r-1}^{j_{r-1}} \varepsilon_{r}$ (à une permutation près), où les $j_{k} \in\{0,1\}$, telle que $\beta \varepsilon$ est un carré dans $K_{0}$, alors $\left\{\varepsilon_{1}, \ldots, \varepsilon_{r-1}, \sqrt{-\varepsilon}\right\}$ est un SFU de $K$.

(2) Dans le cas contraire $\left\{\varepsilon_{1}, \ldots, \varepsilon_{r}\right\}$ est un SFU de $K$.

LEMmE 1. Soient $K=k_{1}\left(\sqrt{-n \varepsilon_{1} \sqrt{2}}\right)$ où $k_{1}=\mathbb{Q}(\sqrt{2})$, $n$ un entier naturel impair sans facteurs carrés et $\varepsilon_{1}$ l'unité fondamentale de $k_{1}$. Alors $\left\{\varepsilon_{1}\right\}$ est un SFU de $K$.

Démonstration. Puisque $\left\{\varepsilon_{1}\right\}$ est un SFU de $k_{1}$ et $n \sqrt{2}$ n'est pas un carré dans $k_{1}$, d'après la proposition $2,\left\{\varepsilon_{1}\right\}$ est un SFU de $K$.

Lemme 2 (voir [3]). Soient $d$ un entier relatif sans facteurs carrés et $\varepsilon=x+y \sqrt{d}$ l'unité fondamentale de $\mathbb{Q}(\sqrt{d})$ où $x$ et $y$ sont des entiers ou 
bien des demi-entiers. On suppose que $\varepsilon$ est de norme 1 . Alors $2(x \pm 1)$ et $2 d(x \pm 1)$ ne sont pas des carrés dans $\mathbb{Q}$.

Remarque 1 . Soit $\varepsilon=x+y \sqrt{d}$ l'unité fondamentale de $\mathbb{Q}(\sqrt{d})$ où $d$ est un entier relatif sans facteurs carrés. On suppose que $\varepsilon$ est de norme 1 . Alors $2 \varepsilon$ est un carré dans $\mathbb{Q}(\sqrt{d})$ si et seulement si $x \pm 1$ est un carré dans $\mathbb{Q}$. En effet,

$$
2 \varepsilon=(a+b \sqrt{d})^{2} \Leftrightarrow\left\{\begin{array}{l}
2 x=a^{2}+d b^{2}, \\
2 y=2 a b .
\end{array}\right.
$$

Comme $x^{2}-d y^{2}=1$, le dernier système est résoluble si et seulement si $x \pm 1$ est un carré dans $\mathbb{Q}$.

Lemme 3 (voir [3]). Soient $p$ un nombre premier impair et $\varepsilon=x+y \sqrt{2 p}$ l'unité fondamentale de $\mathbb{Q}(\sqrt{2 p})$. On suppose que $\varepsilon$ est de norme 1 . Alors $x \pm 1$ est un carré dans $\mathbb{N}$ et $2 \varepsilon$ est un carré dans $\mathbb{Q}(\sqrt{2 p})$.

Lemme 4. Soient $p, q$ deux nombres premiers différents tels que $p \equiv$ $q \equiv-1 \bmod 4,\left(\frac{2}{p}\right)=\left(\frac{2}{q}\right)=-1$, et $\varepsilon=s+t \sqrt{2 p q}$ l'unité fondamentale de $\mathbb{Q}(\sqrt{2 p q})$. Alors $s-1$ est un carré dans $\mathbb{N}$ et $2 \varepsilon$ est un carré dans $\mathbb{Q}(\sqrt{2 p q})$.

Démonstration. Puisque $\varepsilon$ est de norme 1 , on a $(s+1)(s-1)=2 p q t^{2}$, et comme le plus grand commun diviseur de $s+1$ et $s-1$ divise 2 , il existe $\alpha, \beta$ et $\gamma$ dans $\{0,1\}$ tels que $2^{\alpha} p^{\beta} q^{\gamma}(s+1)$ est un carré dans $\mathbb{N}$.

- D'après le lemme $2,2(s+1)$ et $p q(s+1)$ ne sont pas des carrés dans $\mathbb{N}$.

- Si $\sqrt{2 p(s+1)} \in \mathbb{N}$, alors il existe $\left(t_{1}, t_{2}\right) \in \mathbb{Z}^{2}$ tels que

$$
s+1=2 p t_{1}^{2}, \quad s-1=q t_{2}^{2}, \quad t_{1} t_{2}=t ;
$$

ainsi $2=2 p t_{1}^{2}-q t_{2}^{2}$, ce qui implique d'une part que $\left(\frac{2}{q}\right)=\left(\frac{2 p}{q}\right)=\left(\frac{2}{q}\right)\left(\frac{p}{q}\right)$, donc $\left(\frac{p}{q}\right)=1$, et d'autre part que $\left(\frac{2}{p}\right)=\left(\frac{-q}{p}\right)=\left(\frac{-1}{p}\right)\left(\frac{q}{p}\right)$, donc $\left(\frac{q}{p}\right)=1$; ainsi $\left(\frac{p}{q}\right)=\left(\frac{q}{p}\right)$, ce qui est impossible puisque $p \equiv q \equiv-1 \bmod 4$. Et de même on montre que $\sqrt{2 q(s+1)} \notin \mathbb{N}$.

- Si $\sqrt{p(s+1)} \in \mathbb{N}$, alors il existe $\left(t_{1}, t_{2}\right) \in \mathbb{Z}^{2}$ tels que

$$
s+1=p t_{1}^{2}, \quad s-1=2 q t_{2}^{2}, \quad t_{1} t_{2}=t ;
$$

ainsi $2=p t_{1}^{2}-2 q t_{2}^{2}$, ce qui implique d'une part que $\left(\frac{2}{p}\right)=\left(\frac{-2 q}{p}\right)=$ $\left(\frac{-1}{p}\right)\left(\frac{2}{p}\right)\left(\frac{q}{p}\right)$, donc $\left(\frac{q}{p}\right)=-1$, et d'autre part que $\left(\frac{2}{q}\right)=\left(\frac{p}{q}\right) ;$ ainsi $\left(\frac{p}{q}\right)=\left(\frac{q}{p}\right)$, ce qui est impossible puisque $p \equiv q \equiv-1 \bmod 4$. Et de même on montre que $\sqrt{q(s+1)} \notin \mathbb{N}$.

Ainsi $2 p q(s+1)$ est un carré dans $\mathbb{N}$, ce qui montre que $s-1$ est un carré dans $\mathbb{N}$ et ainsi $2 \varepsilon$ est un carré dans $\mathbb{Q}(\sqrt{2 p q})$.

ThÉORÈme 4. Soient $p, q$ deux nombres premiers différents tels que $p \equiv q \equiv-1 \bmod 4,\left(\frac{2}{p}\right)=\left(\frac{2}{q}\right)=-1, K_{0}=\mathbb{Q}(\sqrt{2}, \sqrt{p q}), \varepsilon_{1}\left(\right.$ resp. $\left.\varepsilon_{2}, \varepsilon_{3}\right)$ 
l'unité fondamentale de $k_{1}=\mathbb{Q}(\sqrt{2})$ (resp. $k_{2}=\mathbb{Q}(\sqrt{p q}), k_{3}=\mathbb{Q}(\sqrt{2 p q})$ ) et $F_{1}=K_{0}\left(\sqrt{-\varepsilon_{1} \sqrt{2}}\right)$. Alors $\left\{\varepsilon_{1}, \varepsilon_{2}, \sqrt{\varepsilon_{3}}\right\}$ est un $\mathbf{S F U}$ de $K_{0}$ et de $F_{1}$.

Démonstration. On sait d'après [18] que

$$
h_{2}\left(K_{0}\right)=\frac{1}{4} Q_{K_{0}} h_{2}(2) h_{2}(p q) h_{2}(2 p q),
$$

où $h_{2}\left(K_{0}\right), h_{2}(m)$ sont respectivement la 2-partie du nombre de classes de $K_{0}$ et $\mathbb{Q}(\sqrt{m})$ où $m=2, p q, 2 p q$. Or $h_{2}(2)=h_{2}(p q)=1$ et comme $p \equiv$ $q \equiv 3 \bmod 8$, d'après [12] on a $h_{2}(2 p q)=2$; de plus, $K_{0} / \mathbb{Q}(\sqrt{2 p q})$ est une extension non ramifiée et le 2 -groupe de classes de $\mathbb{Q}(\sqrt{2 p q})$ est cyclique, donc $h_{2}\left(K_{0}\right)=h_{2}(2 p q) / 2=1$; ainsi $Q_{K_{0}}=2$. D'après le lemme $4,2 \varepsilon_{3}$ est un carré dans $k_{3}$, donc un carré dans $K_{0}$, d'où $\sqrt{\varepsilon_{3}} \in K_{0}\left(\right.$ car $\left.\sqrt{2} \in K_{0}\right)$; ainsi en utilisant les résultats de [15] on trouve que $\left\{\varepsilon_{1}, \varepsilon_{2}, \sqrt{\varepsilon_{3}}\right\}$ est un SFU de $K_{0}$.

Montrons que $\left\{\varepsilon_{1}, \varepsilon_{2}, \sqrt{\varepsilon_{3}}\right\}$ est aussi un SFU de $F_{1}$. En effet, soient $\varepsilon_{2}=x+y \sqrt{p q}$ et $\varepsilon_{3}=s+t \sqrt{2 p q}$ et $N_{i}$ la norme de $K_{0}$ sur $k_{i}(i=1,2,3)$; alors d'après la proposition 2 , pour montrer que $\left\{\varepsilon_{1}, \varepsilon_{2}, \sqrt{\varepsilon_{3}}\right\}$ est un SFU de $F_{1}$, on montre que $\eta \varepsilon_{1} \sqrt{2}$ n'est pas un carré dans $K_{0}$, pour $\eta=\varepsilon_{1}^{\prime j_{1}} \varepsilon_{2}^{\prime j_{2}} \varepsilon_{3}^{\prime}$ où $\left\{\varepsilon_{1}^{\prime}, \varepsilon_{2}^{\prime}, \varepsilon_{3}^{\prime}\right\}=\left\{\varepsilon_{1}, \varepsilon_{2}, \sqrt{\varepsilon_{3}}\right\}$ et $j_{1}, j_{2} \in\{0,1\}$. Alors si $\eta=\varepsilon_{1}$, on montre que $\varepsilon_{1}^{2} \sqrt{2}$ n'est pas un carré dans $K_{0}$ : sinon il existe $x \in K_{0}$ tel que $\sqrt{2}=x^{2}$, et comme $N_{2}(\sqrt{2})=N_{2}(x)^{2}=-2$ et $N_{2}(x) \in k_{2}$, il s'ensuit que -2 est un carré dans $k_{2}=\mathbb{Q}(\sqrt{p q})$, ce qui est absurde. Si $\eta=\varepsilon_{2}$, on suppose qu'il existe $x \in K_{0}$ tel que $\varepsilon_{1} \varepsilon_{2} \sqrt{2}=x^{2}$; or $N_{3}\left(\varepsilon_{1}\right)=-1, N_{3}\left(\varepsilon_{2}\right)=1$ et $N_{3}(\sqrt{2})=-2$, donc $N_{3}\left(\varepsilon_{1} \varepsilon_{2} \sqrt{2}\right)=N_{3}(x)^{2}=2$, et puisque $N_{3}(x) \in k_{3}$, donc 2 est un carré dans $k_{3}=\mathbb{Q}(\sqrt{2 p q})$, ce qui est impossible. Si $\eta=\varepsilon_{1} \varepsilon_{2}$, on suppose qu'il existe $x \in K_{0}$ tel que $\varepsilon_{2} \sqrt{2}=x^{2}$; comme $N_{3}\left(\varepsilon_{2}\right)=1$ et $N_{3}(\sqrt{2})=-2$, on a $N_{3}\left(\varepsilon_{2} \sqrt{2}\right)=N_{3}(x)^{2}=-2$, ainsi -2 est un carré dans $k_{3}$, ce qui est impossible. Pour $\eta=\sqrt{\varepsilon_{3}}$ (resp. $\eta=\varepsilon_{1} \sqrt{\varepsilon_{3}}, \varepsilon_{2} \sqrt{\varepsilon_{3}}, \varepsilon_{1} \varepsilon_{2} \sqrt{\varepsilon_{3}}$ ) on voit que $\varepsilon_{1} \sqrt{2 \varepsilon_{3}}$ (resp. $\sqrt{2 \varepsilon_{3}}, \varepsilon_{1} \varepsilon_{2} \sqrt{2 \varepsilon_{3}}, \varepsilon_{2} \sqrt{2 \varepsilon_{3}}$ ) n'est pas un carré dans $K_{0}$, sinon en utilisant la norme de $K_{0}$ sur $k_{2}$ on trouve que $\sqrt{ \pm 2} \in k_{2}$, ce qui est absurde. Cela prouve que $\left\{\varepsilon_{1}, \varepsilon_{2}, \sqrt{\varepsilon_{3}}\right\}$ est un SFU de $F_{1}$.

THÉORÈme 5. Soient $p, q$ deux nombres premiers différents tel que $p \equiv$ $q \equiv 5 \bmod 8, K_{0}=\mathbb{Q}(\sqrt{2}, \sqrt{p q}), \varepsilon_{1}$ (resp. $\left.\varepsilon_{2}, \varepsilon_{3}\right)$ l'unité fondamentale de $k_{1}=\mathbb{Q}(\sqrt{2})\left(\right.$ resp. $\left.k_{2}=\mathbb{Q}(\sqrt{p q}), k_{3}=\mathbb{Q}(\sqrt{2 p q})\right)$ et $F_{1}=K_{0}\left(\sqrt{-\varepsilon_{1} \sqrt{2}}\right)$. Alors $K_{0}$ et $F_{1}$ ont même $\mathbf{S F U}$ qui est l'un des systèmes $\left\{\varepsilon_{1}, \varepsilon_{2}, \varepsilon_{3}\right\}$ ou $\left\{\sqrt{\varepsilon_{1} \varepsilon_{2} \varepsilon_{3}}, \varepsilon_{2}, \varepsilon_{3}\right\}$.

Démonstration. Comme $\left(\frac{2}{p}\right)=\left(\frac{2}{q}\right)=-1$, l'unité $\varepsilon_{3}$ est de norme -1 , et $\varepsilon_{1}$ l'est aussi; ainsi, si $\varepsilon_{2}$ est de norme 1 , alors comme $\sqrt{\varepsilon_{2}} \notin K_{0}$ (voir [5, lemme 1]), d'après les résultats de [15], $\left\{\varepsilon_{1}, \varepsilon_{2}, \varepsilon_{3}\right\}$ est un SFU de $K_{0}$. Et si $\varepsilon_{2}$ est de norme -1 , alors toujours d'après les résultats de [15], $\left\{\varepsilon_{1}, \varepsilon_{2}, \varepsilon_{3}\right\}$ 
est un SFU de $K_{0}$ si $\sqrt{\varepsilon_{1} \varepsilon_{2} \varepsilon_{3}} \notin K_{0}$, et $\left\{\sqrt{\varepsilon_{1} \varepsilon_{2} \varepsilon_{3}}, \varepsilon_{2}, \varepsilon_{3}\right\}$ est un SFU de $K_{0}$ dans le cas contraire.

Montrons que $K_{0}$ et $F_{1}$ ont même SFU. En effet, soient $\varepsilon_{2}=x+y \sqrt{p q}$ et $\varepsilon_{3}=s+t \sqrt{2 p q}$ et $N_{i}$ la norme de $K_{0}$ sur $k_{i}(i=1,2,3)$.

Si $\left\{\varepsilon_{1}, \varepsilon_{2}, \varepsilon_{3}\right\}$ est un SFU de $K_{0}$ (dans ce cas $\varepsilon_{1}, \varepsilon_{3}$ sont de norme -1 et $\varepsilon_{2}$ est de norme \pm 1 ), alors d'après la proposition 2 , pour montrer que $\left\{\varepsilon_{1}, \varepsilon_{2}, \varepsilon_{3}\right\}$ est un SFU de $F_{1}$, on montre que $\eta \varepsilon_{1} \sqrt{2}$ n'est pas un carré dans $K_{0}$ pour $\eta=\varepsilon_{1}^{\prime j_{1}} \varepsilon_{2}^{\prime j_{2}} \varepsilon_{3}^{\prime}$ où $\left\{\varepsilon_{1}^{\prime}, \varepsilon_{2}^{\prime}, \varepsilon_{3}^{\prime}\right\}=\left\{\varepsilon_{1}, \varepsilon_{2}, \varepsilon_{3}\right\}$ et $j_{1}, j_{2} \in\{0,1\}$. Alors si $\eta=\varepsilon_{1}$, on montre que $\varepsilon_{1}^{2} \sqrt{2}$ n'est pas un carré dans $K_{0}$ : sinon il existe $x \in K_{0}$ tel que $\sqrt{2}=x^{2}$, et comme $N_{2}(\sqrt{2})=N_{2}(x)^{2}=-2$ et $N_{2}(x) \in k_{2}$, le nombre -2 est un carré dans $k_{2}=\mathbb{Q}(\sqrt{p q})$, ce qui est absurde. Si $\eta=\varepsilon_{2}$, on suppose qu'il existe $x \in K_{0}$ tel que $\varepsilon_{1} \varepsilon_{2} \sqrt{2}=x^{2}$; or $N_{3}\left(\varepsilon_{1}\right)=-1$, $N_{3}\left(\varepsilon_{2}\right)= \pm 1$ et $N_{3}(\sqrt{2})=-2$, donc $N_{3}\left(\varepsilon_{1} \varepsilon_{2} \sqrt{2}\right)=N_{3}(x)^{2}= \pm 2$, et puisque $N_{3}(x) \in k_{3}$, il vient que \pm 2 est un carré dans $k_{3}=\mathbb{Q}(\sqrt{2 p q})$, ce qui est impossible. Si $\eta=\varepsilon_{3}$, supposons qu'il existe $x \in K_{0}$ tel que $\varepsilon_{1} \varepsilon_{3} \sqrt{2}=x^{2}$; comme $N_{2}\left(\varepsilon_{1}\right)=-1, N_{2}\left(\varepsilon_{3}\right)=-1$ et $N_{2}(\sqrt{2})=-2$, on a $N_{2}\left(\varepsilon_{1} \varepsilon_{3} \sqrt{2}\right)=$ $N_{2}(x)^{2}=-2$; ainsi -2 est un carré dans $k_{2}=\mathbb{Q}(\sqrt{p q})$, ce qui n'est pas le cas. Si $\eta=\varepsilon_{2} \varepsilon_{3}$, on suppose qu'il existe $x \in K_{0}$ tel que $\varepsilon_{1} \varepsilon_{2} \varepsilon_{3} \sqrt{2}=x^{2}$; comme $N_{2}\left(\varepsilon_{1}\right)=-1, N_{2}\left(\varepsilon_{2}\right)=\varepsilon_{2}^{2}, N_{2}\left(\varepsilon_{3}\right)=-1$ et $N_{2}(\sqrt{2})=-2$, on a $N_{2}\left(\varepsilon_{1} \varepsilon_{2} \varepsilon_{3} \sqrt{2}\right)=N_{2}(x)^{2}=-2 \varepsilon_{2}^{2}$; ainsi -2 est un carré dans $k_{2}=\mathbb{Q}(\sqrt{p q})$, ce qui est impossible. Et par des raisonnements analogues on démontre les autres cas.

Si $\left\{\sqrt{\varepsilon_{1} \varepsilon_{2} \varepsilon_{3}}, \varepsilon_{2}, \varepsilon_{3}\right\}$ est un SFU de $K_{0}$ (dans ce cas $\varepsilon_{1}$, $\varepsilon_{2}$ et $\varepsilon_{3}$ sont de norme -1$)$, alors d'après la proposition 2 , pour montrer que $\left\{\sqrt{\varepsilon_{1} \varepsilon_{2} \varepsilon_{3}}, \varepsilon_{2}, \varepsilon_{3}\right\}$ est un SFU de $F_{1}$, on montre que $\eta \varepsilon_{1} \sqrt{2}$ n'est pas un carré dans $K_{0}$ pour $\eta=\varepsilon_{1}^{\prime j_{1}} \varepsilon_{2}^{\prime j_{2}} \varepsilon_{3}^{\prime}$ où $\left\{\varepsilon_{1}^{\prime}, \varepsilon_{2}^{\prime}, \varepsilon_{3}^{\prime}\right\}=\left\{\sqrt{\varepsilon_{1} \varepsilon_{2} \varepsilon_{3}}, \varepsilon_{2}, \varepsilon_{3}\right\}$ et $j_{1}, j_{2} \in\{0,1\}$. Alors si $\eta=\sqrt{\varepsilon_{1} \varepsilon_{2} \varepsilon_{3}}$, on montre que $\varepsilon_{1} \sqrt{2 \varepsilon_{1} \varepsilon_{2} \varepsilon_{3}}$ n'est pas un carré dans $K_{0}:$ sinon il existe $x \in K_{0}$ tel que $\varepsilon_{1} \sqrt{2 \varepsilon_{1} \varepsilon_{2} \varepsilon_{3}}=x^{2}$, et comme $N_{2}\left(\varepsilon_{1}\right)=N_{2}\left(\varepsilon_{3}\right)=-1$, $N_{2}\left(\varepsilon_{2}\right)=\varepsilon_{2}^{2}$ et $N_{2}(\sqrt{2})=-2$, on a $N_{2}\left(\varepsilon_{1} \sqrt{2 \varepsilon_{1} \varepsilon_{2} \varepsilon_{3}}\right)=N_{2}(x)^{2}=\mp 2 \varepsilon_{2}$; ainsi $\mp 2 \varepsilon_{2}$ est un carré dans $k_{2}=\mathbb{Q}(\sqrt{p q})$, donc on a une contradiction. Pour $\eta=$ $\varepsilon_{2}, \varepsilon_{3}, \varepsilon_{2} \varepsilon_{3}$, on utilise le même raisonnement que dans le cas où $\left\{\varepsilon_{1}, \varepsilon_{2}, \varepsilon_{3}\right\}$ est un SFU de $K_{0}$. Si $\eta=\varepsilon_{2} \sqrt{\varepsilon_{1} \varepsilon_{2} \varepsilon_{3}}$, supposons qu'il existe $x \in K_{0}$ tel que $\varepsilon_{1} \varepsilon_{2} \sqrt{2 \varepsilon_{1} \varepsilon_{2} \varepsilon_{3}}=x^{2}$; alors comme $N_{3}\left(\varepsilon_{1} \varepsilon_{2} \sqrt{2 \varepsilon_{1} \varepsilon_{2} \varepsilon_{3}}\right)=N_{3}(x)^{2}= \pm 2 \varepsilon_{3}$, on trouve que $\pm 2 \varepsilon_{3}$ est un carré dans $k_{3}=\mathbb{Q}(\sqrt{2 p q})$, ce qui est absurde. Si $\eta=\varepsilon_{3} \sqrt{\varepsilon_{1} \varepsilon_{2} \varepsilon_{3}}$, supposons qu'il existe $x \in K_{0}$ tel que $\varepsilon_{1} \varepsilon_{3} \sqrt{2 \varepsilon_{1} \varepsilon_{2} \varepsilon_{3}}=x^{2}$; alors comme $N_{2}\left(\varepsilon_{1} \varepsilon_{3} \sqrt{2 \varepsilon_{1} \varepsilon_{2} \varepsilon_{3}}\right)=N_{2}(x)^{2}= \pm 2 \varepsilon_{2}$, on trouve que $\pm 2 \varepsilon_{2}$ est un carré dans $k_{2}$, donc un carré dans $K_{0}$, ce qui est absurde. Enfin si $\eta=$ $\varepsilon_{2} \varepsilon_{3} \sqrt{\varepsilon_{1} \varepsilon_{2} \varepsilon_{3}}$, supposons qu'il existe $x \in K_{0}$ tel que $\varepsilon_{1} \varepsilon_{2} \varepsilon_{3} \sqrt{2 \varepsilon_{1} \varepsilon_{2} \varepsilon_{3}}=x^{2}$; alors comme $N_{2}\left(\varepsilon_{1} \varepsilon_{2} \varepsilon_{3} \sqrt{2 \varepsilon_{1} \varepsilon_{2} \varepsilon_{3}}\right)=N_{2}(x)^{2}= \pm 2 \varepsilon_{2}^{3}$, on trouve que $\pm 2 \varepsilon_{2}$ est un carré dans $K_{0}$, ce qui n'est pas le cas. Ceci achève la démonstration. 
ThÉORÈme 6 . Soient $p$ et $q$ deux nombres premiers impairs différents tel que $p \equiv 1 \bmod 4, K_{0}=\mathbb{Q}(\sqrt{2}, \sqrt{p}), \varepsilon_{1}$ (resp. $\left.\varepsilon_{2}, \varepsilon_{3}\right)$ l'unité fondamentale de $k_{1}=\mathbb{Q}(\sqrt{2})\left(\right.$ resp. $\left.k_{2}=\mathbb{Q}(\sqrt{p}), k_{3}=\mathbb{Q}(\sqrt{2 p})\right)$ et $F_{2}=K_{0}\left(\sqrt{-q \varepsilon_{1} \sqrt{2}}\right)$. Alors on $a$ :

(i) Si $\varepsilon_{3}$ est de norme 1 , alors $\left\{\varepsilon_{1}, \varepsilon_{2}, \sqrt{\varepsilon_{3}}\right\}$ est un $\mathbf{S F U}$ de $K_{0}$ et de $F_{2}$.

(ii) Sinon, $\left\{\sqrt{\varepsilon_{1} \varepsilon_{2} \varepsilon_{3}}, \varepsilon_{2}, \varepsilon_{3}\right\}$ est un $\mathbf{S F U}$ de $K_{0}$ et de $F_{2}$.

Démonstration. D'après [18], on a $h_{2}\left(K_{0}\right)=\frac{1}{4} Q_{K_{0}} h_{2}(2) h_{2}(p) h_{2}(2 p)$, où $h_{2}\left(K_{0}\right), h_{2}(m)$ sont respectivement la 2-partie du nombre de classes de $K_{0}$ et de $\mathbb{Q}(\sqrt{m})$ avec $m=2, p, 2 p$. Or d'après [4] on a $h_{2}\left(K_{0}\right)=\frac{1}{2} h_{2}(2 p)$ et comme $h_{2}(2)=h_{2}(p)=1$, il vient que $Q_{K_{0}}=2$.

(i) $\mathrm{Si} \varepsilon_{3}$ est de norme 1 , alors d'après le lemme $3,2 \varepsilon_{3}$ est un carré dans $k_{3}$, et comme 2 est un carré dans $K_{0}$, il s'ensuit que $\varepsilon_{3}$ est un carré dans $K_{0}$, et puisque $\varepsilon_{1}$ et $\varepsilon_{2}$ sont de norme -1 et $Q_{K_{0}}=2$, les résultats de [15] impliquent que $\left\{\varepsilon_{1}, \varepsilon_{2}, \sqrt{\varepsilon_{3}}\right\}$ est un SFU de $K_{0}$.

Montrons que $\left\{\varepsilon_{1}, \varepsilon_{2}, \sqrt{\varepsilon_{3}}\right\}$ est aussi un SFU de $F_{2}$. En effet, soit $N_{i}$ la norme de $K_{0}$ sur $k_{i}(i=1,2,3)$. En utilisant la proposition 2, il suffit de montrer que $q \eta \varepsilon_{1} \sqrt{2}$ n'est pas un carré dans $K_{0}$ pour $\eta=\varepsilon_{1}^{\prime j_{1}} \varepsilon_{2}^{\prime j_{2}} \varepsilon_{3}^{\prime}$ où $\left\{\varepsilon_{1}^{\prime}, \varepsilon_{2}^{\prime}, \varepsilon_{3}^{\prime}\right\}=\left\{\varepsilon_{1}, \varepsilon_{2}, \sqrt{\varepsilon_{3}}\right\}$ et $j_{1}, j_{2} \in\{0,1\}$. Alors si $\eta=\varepsilon_{1}$, on montre que $q \varepsilon_{1}^{2} \sqrt{2}$ n'est pas un carré dans $K_{0}$ : sinon il existe $x \in K_{0}$ tel que $q \sqrt{2}=x^{2}$, ainsi $N_{2}(q \sqrt{2})=N_{2}(x)^{2}=-2 q^{2}$, et comme $N_{2}(x) \in k_{2}$, il vient que -2 est un carré dans $k_{2}=\mathbb{Q}(\sqrt{p})$, ce qui est absurde. Si $\eta=\varepsilon_{2}$, supposons qu'il existe $x \in K_{0}$ tel que $q \varepsilon_{1} \varepsilon_{2} \sqrt{2}=x^{2}$, donc $N_{3}\left(q \varepsilon_{1} \varepsilon_{2} \sqrt{2}\right)=N_{3}(x)^{2}=-2 q^{2}$; ainsi -2 est un carré dans $k_{3}=\mathbb{Q}(\sqrt{2 p})$, ce qui est impossible. Si $\eta=\sqrt{\varepsilon_{3}}$, supposons qu'il existe $x \in K_{0}$ tel que $q \varepsilon_{1} \sqrt{2 \varepsilon_{3}}=x^{2}$, donc $N_{2}\left(q \varepsilon_{1} \sqrt{2 \varepsilon_{3}}\right)=$ $N_{2}(x)^{2}=\mp 2 q^{2}$; ainsi $\mp 2$ est un carré dans $k_{2}=\mathbb{Q}(\sqrt{p})$, ce qui n'est pas le cas. Et de même on démontre les autres cas.

(ii) $\mathrm{Si} \varepsilon_{3}$ est de norme -1 , alors comme $\varepsilon_{1}$ et $\varepsilon_{2}$ sont de norme -1 et $Q_{K_{0}}=2$, en utilisant les résultats de [15] on trouve que $\left\{\sqrt{\varepsilon_{1} \varepsilon_{2} \varepsilon_{3}}, \varepsilon_{2}, \varepsilon_{3}\right\}$ est un SFU de $K_{0}$. En particulier, on a ceci si $\left(\frac{2}{p}\right)=-1$.

Le système $\left\{\sqrt{\varepsilon_{1} \varepsilon_{2} \varepsilon_{3}}, \varepsilon_{2}, \varepsilon_{3}\right\}$ est aussi un SFU de $F_{2}$. En effet, soit $N_{i}$ la norme de $K_{0}$ sur $k_{i}(i=1,2,3)$; alors d'après la proposition 2, il suffit de montrer que $q \eta \varepsilon_{1} \sqrt{2}$ n'est pas un carré dans $K_{0}$ pour $\eta=\varepsilon_{1}^{\prime j_{1}} \varepsilon_{2}^{\prime j_{2}} \varepsilon_{3}^{\prime}$ où $\left\{\varepsilon_{1}^{\prime}, \varepsilon_{2}^{\prime}, \varepsilon_{3}^{\prime}\right\}=\left\{\sqrt{\varepsilon_{1} \varepsilon_{2} \varepsilon_{3}}, \varepsilon_{2}, \varepsilon_{3}\right\}$ et $j_{1}, j_{2} \in\{0,1\}$. Alors si $\eta=\sqrt{\varepsilon_{1} \varepsilon_{2} \varepsilon_{3}}$, montrons que $q \varepsilon_{1} \sqrt{2 \varepsilon_{1} \varepsilon_{2} \varepsilon_{3}}$ n'est pas un carré dans $K_{0}$ : sinon il existe $x \in K_{0}$ tel que $q \varepsilon_{1} \sqrt{2 \varepsilon_{1} \varepsilon_{2} \varepsilon_{3}}=x^{2}$, ainsi $N_{2}\left(q \varepsilon_{1} \sqrt{2 \varepsilon_{1} \varepsilon_{2} \varepsilon_{3}}\right)=N_{2}(x)^{2}=\mp 2 q^{2} \varepsilon_{2}$, et puisque $N_{2}(x) \in k_{2}$, on en déduit que $\mp 2 \varepsilon_{2}$ est un carré dans $k_{2}$, ce qui est absurde. Si $\eta=\varepsilon_{2}$, supposons qu'il existe $x$ dans $K_{0}$ tel que $q \varepsilon_{1} \varepsilon_{2} \sqrt{2}=x^{2}$; ainsi $N_{3}\left(q \varepsilon_{1} \varepsilon_{2} \sqrt{2}\right)=N_{3}(x)^{2}=-2 q^{2}$, et puisque $N_{3}(x) \in k_{3}$, il vient que -2 est un carré dans $k_{3}$, ce qui est impossible. Si $\eta=\varepsilon_{3}$, supposons qu'il existe $x$ dans $K_{0}$ tel que $q \varepsilon_{1} \varepsilon_{3} \sqrt{2}=x^{2}$; ainsi $N_{2}\left(q \varepsilon_{1} \varepsilon_{3} \sqrt{2}\right)=N_{2}(x)^{2}=-2 q^{2}$, 
donc -2 est un carré dans $k_{2}$, ce qui n'est pas le cas. Si $\eta=\varepsilon_{2} \sqrt{\varepsilon_{1} \varepsilon_{2} \varepsilon_{3}}$, supposons que $q \varepsilon_{1} \varepsilon_{2} \sqrt{2 \varepsilon_{1} \varepsilon_{2} \varepsilon_{3}}$ est un carré dans $K_{0}$, donc il existe $x \in K_{0}$ tel que $q \varepsilon_{1} \varepsilon_{2} \sqrt{2 \varepsilon_{1} \varepsilon_{2} \varepsilon_{3}}=x^{2}$; comme $N_{3}\left(\varepsilon_{1}\right)=N_{3}\left(\varepsilon_{2}\right)=-1$ et $N_{3}\left(\varepsilon_{3}\right)=\varepsilon_{3}^{2}$, alors $\left.N_{3}\left(q \varepsilon_{1} \varepsilon_{2} \sqrt{2 \varepsilon_{1} \varepsilon_{2} \varepsilon_{3}}\right)= \pm N_{3}\left(q \varepsilon_{1} \varepsilon_{2}\right) \sqrt{N_{3}\left(2 \varepsilon_{1} \varepsilon_{2} \varepsilon_{3}\right.}\right)= \pm 2 q^{2} \varepsilon_{3}=N_{3}(x)^{2}$, ainsi $\pm 2 \varepsilon_{3}$ est un carré dans $k_{3}$, ce qui est absurde. Et de même on démontre les autres cas.

\section{Capitulation des 2-classes d'idéaux de $K$ et structure de $G$}

Proposition 3 (voir [17] ou [16]). Soient $L / M$ une extension biquadratique normale de groupe de Galois de type $(2,2)$, et $L_{1}, L_{2}, L_{3}$ ses sousextensions quadratiques. Alors

$$
h(L)=\frac{2^{d-\kappa-2-v} q(L) h\left(L_{1}\right) h\left(L_{2}\right) h\left(L_{3}\right)}{h(M)^{2}},
$$

où $q(L)=\left[E_{L}: E_{1} E_{2} E_{3}\right]$ est l'indice des unités de $L / M, d$ le nombre des premiers infinis de $M$ qui se ramifient dans $L / M, \kappa$ est le $\mathbb{Z}$-rang du groupe $E_{M}$ des unités de $M$, et $v=0$ sauf si $L \subseteq M\left(\sqrt{E_{M}}\right)$ où $v=1$.

Dans toute la suite, soient $p, q$ deux nombres premiers différents tels que $p \equiv q \equiv \pm 1 \bmod 4$ et $\left(\frac{2}{p}\right)=\left(\frac{2}{q}\right)=-1, K=k\left(\sqrt{-p q \varepsilon_{1} \sqrt{2}}\right)$ où $\varepsilon_{1}$ est l'unité fondamentale de $k=\mathbb{Q}(\sqrt{2}), K_{2}^{(1)}$ le 2-corps de classes de Hilbert de $K$, $K_{2}^{(2)}$ le 2-corps de classes de Hilbert de $K_{2}^{(1)}$ et $G$ le groupe de Galois de $K_{2}^{(2)} / K$. Alors, d'après E. Brown et C. J. Parry [7], $C_{2, K} \simeq \mathbb{Z} / 2 \mathbb{Z} \times \mathbb{Z} / 2 \mathbb{Z}$, d'où $K_{2}^{(1)} / K$ contient trois extensions $F_{i} / K(i=1,2,3)$, la tour des 2-corps de classes de Hilbert de $K$ s'arrête en $K_{2}^{(2)}$ et on a :

- Si $p \equiv q \equiv-1 \bmod 4$, alors $K_{2}^{(1)}=K^{(*)}=K(\sqrt{-p}, \sqrt{-q})$ de souscorps quadratiques sur $K: F_{1}=K(\sqrt{p q}), F_{2}=K(\sqrt{-p})$ et $F_{3}=$ $K(\sqrt{-q})$.

- Si $p \equiv q \equiv 1 \bmod 4$, alors $K_{2}^{(1)}=K^{(*)}=K(\sqrt{p}, \sqrt{q})$ de sous-corps quadratiques sur $K: F_{1}=K(\sqrt{p q}), F_{2}=K(\sqrt{p})$ et $F_{3}=K(\sqrt{q})$.

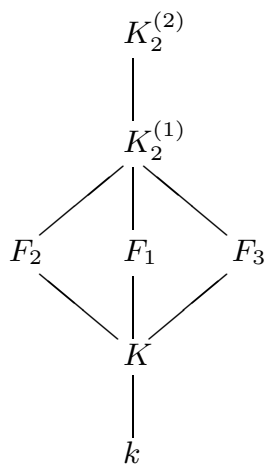


On va faire une étude du problème de la capitulation des 2-classes d'idéaux de $K$ dans les différentes sous-extensions quadratiques $F_{i} / K$ de $K_{2}^{(1)} / K$ (voir le diagramme), et par suite nous déterminerons la structure de $G$.

\subsection{Cas où $p \equiv q \equiv-1 \bmod 4$}

THÉORÈme 7 . Soient $K=k\left(\sqrt{-p q \varepsilon_{1} \sqrt{2}}\right)$ où $\varepsilon_{1}$ est l'unité fondamentale de $k=\mathbb{Q}(\sqrt{2}), p, q$ deux nombres premiers différents tels que $p \equiv q \equiv$ $-1 \bmod 4$ et $\left(\frac{2}{p}\right)=\left(\frac{2}{q}\right)=-1, F_{1}=K(\sqrt{p q}), F_{2}=K(\sqrt{-p})$ et $F_{3}=$ $K(\sqrt{-q})$. Alors $K_{2}^{(2)}=K_{2}^{(1)}, G=\operatorname{Gal}\left(K_{2}^{(2)} / K\right)$ est abélien et les quatre classes de $C_{2, K}$ capitulent dans chacune des extensions $F_{i} / K(i=1,2,3)$.

Démonstration. Comme $F_{1} / k$ est une extension biquadratique normale de groupe de Galois de type $(2,2)$, de sous-extensions quadratiques $K, K^{\prime}=$ $k\left(\sqrt{-\varepsilon_{1} \sqrt{2}}\right)$ et $K_{0}=\mathbb{Q}(\sqrt{2}, \sqrt{p q})$, la proposition 3 montre que

$$
h_{2}\left(F_{1}\right)=\frac{2^{2-1-2-0} q\left(F_{1}\right) h_{2}(K) h_{2}\left(K^{\prime}\right) h_{2}\left(K_{0}\right)}{h_{2}(k)^{2}} .
$$

Et puisque $h_{2}(k)=1, h_{2}(K)=4$, d'après [6] on a $h_{2}\left(K^{\prime}\right)=1$, et comme $p \equiv q \equiv 3 \bmod 8$, d'après $[12]$ on a $h_{2}(2 p q)=2$, et comme $K_{0} / \mathbb{Q}(\sqrt{2 p q})$ est une extension non ramifiée et le 2 -groupe de classes de $\mathbb{Q}(\sqrt{2 p q})$ est cyclique, d'après [8] on a $h_{2}\left(K_{0}\right)=h_{2}(2 p q) / 2=1$, ainsi $h_{2}\left(F_{1}\right)=2 q\left(F_{1}\right)$.

Montrons que $q\left(F_{1}\right)=1$. En effet, soient $\varepsilon_{1}$ l'unité fondamentale de $\mathbb{Q}(\sqrt{2}), \varepsilon_{2}$ l'unité fondamentale de $\mathbb{Q}(\sqrt{p q})$ et $\varepsilon_{3}$ l'unité fondamentale de $\mathbb{Q}(\sqrt{2 p q})$; alors d'après le théorème $4,\left\{\varepsilon_{1}, \varepsilon_{2}, \sqrt{\varepsilon_{3}}\right\}$ est un $\mathbf{S F U}$ de $K_{0}$ et de $F_{1}=K(\sqrt{p q})=K_{0}\left(\sqrt{-\varepsilon_{1} \sqrt{2}}\right)$, et comme $\left\{\varepsilon_{1}\right\}$ est un SFU de $K$ et $K^{\prime}$, on trouve que $E_{K} E_{K^{\prime}} E_{K_{0}}=E_{K_{0}}$, ainsi $q\left(F_{1}\right)=\left[E_{F_{1}}: E_{K_{0}}\right]=1$, ainsi $C_{2, F_{1}}$ est cyclique d'ordre 2 , et comme $K_{2}^{(1)} / F_{1}$ est une extension non ramifiée, $F_{1}$ et $K_{2}^{(1)}$ ont même 2-corps de classes de Hilbert, à savoir $K_{2}^{(2)}$; or $h_{2}\left(F_{1}\right)=2$, donc $K_{2}^{(2)}=K_{2}^{(1)}$, ainsi $G$ est abélien $(\simeq \mathbb{Z} / 2 \mathbb{Z} \times \mathbb{Z} / 2 \mathbb{Z})$ et les quatre classes de $C_{2, K}$ capitulent dans chacune des extensions $F_{i} / K(i=1,2,3)$.

4.2. Cas où $p \equiv q \equiv 1 \bmod 4$

4.2.1. Capitulation dans $F_{1} / K$. Soient $K_{0}=\mathbb{Q}(\sqrt{2}, \sqrt{p q}), \varepsilon_{1}$ (resp. $\varepsilon_{2}$, $\left.\varepsilon_{3}\right)$ l'unité fondamentale de $\mathbb{Q}(\sqrt{2})$ (resp. $\mathbb{Q}(\sqrt{p q}), \mathbb{Q}(\sqrt{2 p q})$ ). Alors $F_{1}=$ $K(\sqrt{p q})=K_{0}\left(\sqrt{-\varepsilon_{1} \sqrt{2}}\right)$ et d'après le théorème $5, K_{0}$ et $F_{1}$ ont même SFU qui est l'un des systèmes $\left\{\varepsilon_{1}, \varepsilon_{2}, \varepsilon_{3}\right\}$ ou $\left\{\sqrt{\varepsilon_{1} \varepsilon_{2} \varepsilon_{3}}, \varepsilon_{2}, \varepsilon_{3}\right\}$.

Si $Q_{K_{0}}=1$, donc $\left\{\varepsilon_{1}, \varepsilon_{2}, \varepsilon_{3}\right\}$ est SFU de $F_{1}$, alors $N_{F_{1} / K}\left(E_{F_{1}}\right) \neq E_{K}$. D'où d'après le théorème 1 , les quatres classes de $C_{2, K}$ capitulent dans $F_{1}$ $\left(\left|\operatorname{ker} j_{1}\right|=4\right)$. 
Si $Q_{K_{0}}=2,\left\{\sqrt{\varepsilon_{1} \varepsilon_{2} \varepsilon_{3}}, \varepsilon_{2}, \varepsilon_{3}\right\}$ est un SFU de $F_{1}$, ainsi $N_{F_{1} / K}\left(\sqrt{\varepsilon_{1} \varepsilon_{2} \varepsilon_{3}}\right)=$ $\pm \varepsilon_{1}$, par suite $N_{F_{1} / K}\left(E_{F_{1}}\right)=E_{K}$. D'où d'après le théorème 1 , deux classes seulement de $C_{2, K}$ capitulent dans $F_{1}$, ainsi $\left|\operatorname{ker} j_{1}\right|=2$.

4.2.2. Capitulation dans $F_{i} / K, i=2,3$. Soient $K_{0}^{\prime}=\mathbb{Q}(\sqrt{2}, \sqrt{p}), \varepsilon_{1}$ (resp. $\left.\eta_{2}, \eta_{3}\right)$ l'unité fondamentale de $\mathbb{Q}(\sqrt{2})$ (resp. $\mathbb{Q}(\sqrt{p}), \mathbb{Q}(\sqrt{2 p})$ ). Alors $F_{2}=K(\sqrt{p})=K_{0}^{\prime}\left(\sqrt{-q \varepsilon_{1} \sqrt{2}}\right)$ et d'après le théorème $6,\left\{\sqrt{\varepsilon_{1} \eta_{2} \eta_{3}}, \eta_{2}, \eta_{3}\right\}$ est un SFU de $F_{2}$.

On a $N_{F_{2} / K}\left(\sqrt{\varepsilon_{1} \eta_{2} \eta_{3}}\right)= \pm \varepsilon_{1}$, ainsi $N_{F_{2} / K}\left(E_{F_{2}}\right)=E_{K}$. D'où d'après le théorème 1 , deux classes seulement de $C_{2, K}$ capitulent dans $F_{2}$, et de même pour $F_{3}$, car $p$ et $q$ jouent des rôles symétriques; ainsi $\left|\operatorname{ker} j_{2}\right|=\left|\operatorname{ker} j_{3}\right|=2$.

Proposition 4. Soient $K=k\left(\sqrt{-p q \varepsilon_{1} \sqrt{2}}\right)$ où $\varepsilon_{1}$ est l'unité fondamentale de $k=\mathbb{Q}(\sqrt{2})$ et $\mathcal{P}$ l'idéal premier au-dessus de $p$ dans $K$. Alors la classe $[\mathcal{P}]$ de $\mathcal{P}$ est d'ordre 2 . De plus, $\mathcal{P}$ capitule dans $F_{2}=K(\sqrt{p})$.

Démonstration. La classe $[\mathcal{P}]$ de $\mathcal{P}$ est d'ordre 2 : en effet, comme $p$ est inerte dans $k / \mathbb{Q}$ et $p$ se ramifie dans $K / \mathbb{Q}$, il existe $\mathcal{P}$ un idéal premier de $K$ tel que $\mathcal{P}^{2}=(p)$. On suppose que $\mathcal{P}=(\alpha)$ pour un certain $\alpha$ dans $K$, ce qui est équivalent à $\left(\alpha^{2}\right)=(p)$ dans $K$. Il existe donc $\varepsilon$ une unité de $K$ telle que $p \varepsilon=\alpha^{2}$; or il existe $a$ et $b$ dans $k$ tel que $\alpha=a+b \sqrt{-p q \varepsilon_{1} \sqrt{2}}$, ainsi $p \varepsilon=a^{2}-p q \varepsilon_{1} b^{2} \sqrt{2}+2 a b \sqrt{-p q \varepsilon_{1} \sqrt{2}}$, et comme $\left\{\varepsilon_{1}\right\}$ est SFU de $K$ et $i=\sqrt{-1} \notin K$, on a $p \varepsilon \in k$ et par suite $a=0$ ou $b=0$. Si $b=0$, alors $p \varepsilon=a^{2}$; ainsi, si $\varepsilon$ est de norme 1 (la norme dans $k / \mathbb{Q}$ ), $p$ sera norme dans $k / \mathbb{Q}$, ce qui n'est pas le cas car $\left(\frac{2}{p}\right)=-1$; si $\varepsilon$ est de norme -1 on trouve que -1 est un carré dans $\mathbb{Q}$, ce qui est impossible, et de même, si $a=0$ on trouve que \pm 2 est un carré dans $\mathbb{Q}$. Donc la classe de $\mathcal{P}$ est d'ordre 2 .

Montrons que $\mathcal{P}$ capitule dans $K(\sqrt{p})$. Comme dans le cas précédent, le problème est de chercher $\beta$ dans $K(\sqrt{p})$ tel que $\left(\beta^{2}\right)=(p)$ dans $K(\sqrt{p})$; or ceci est vérifié en prenant $\beta=\sqrt{p}$. Ainsi $\mathcal{P}$ capitule dans $F_{2}=K(\sqrt{p})$.

Proposition 5. Soient $K=k\left(\sqrt{-p q \varepsilon_{1} \sqrt{2}}\right)$ où $\varepsilon_{1}$ est l'unité fondamentale de $k=\mathbb{Q}(\sqrt{2})$ et $\mathcal{Q}$ l'idéal premier au-dessus de $q$ dans $K$. Alors la classe $[\mathcal{Q}]$ est d'ordre 2 . De plus, $\mathcal{Q}$ capitule dans $F_{3}=K(\sqrt{q})$.

Démonstration. Même démonstration que pour la proposition 4.

Proposition 6. Soient $K=k\left(\sqrt{-p q \varepsilon_{1} \sqrt{2}}\right)$ où $\varepsilon_{1}$ est l'unité fondamentale de $k=\mathbb{Q}(\sqrt{2}), \mathcal{P}$ l'idéal premier de $K$ au-dessus de $p$ et $\mathcal{Q}$ celui au-dessus de $q$. Alors la classe $[\mathcal{P} \mathcal{Q}]$ est d'ordre 2 dans $K$ et $C_{2, K}$ le 2-groupe de classes de $K$ est engendré par les classes $[\mathcal{P}]$ et $[\mathcal{Q}]$. De plus, $\mathcal{P} \mathcal{Q}$ capitule dans $K(\sqrt{p q})$.

Démonstration. La classe de $\mathcal{P} \mathcal{Q}$ est d'ordre 2 : sinon il existe $\alpha$ dans $K$ tel que $\mathcal{P} \mathcal{Q}=(\alpha)$, il existe donc $\varepsilon$ une unité de $K$ telle que $p q \varepsilon=\alpha^{2}$; 
or il existe $a$ et $b$ dans $k$ tel que $\alpha=a+b \sqrt{-p q \varepsilon_{1} \sqrt{2}}$, ainsi $p q \varepsilon=a^{2}-$ $p q \varepsilon_{1} b^{2} \sqrt{2}+2 a b \sqrt{-p q \varepsilon_{1} \sqrt{2}}$, et comme $\left\{\varepsilon_{1}\right\}$ est SFU de $K$ et $i=\sqrt{-1} \notin K$, on a $p q \varepsilon \in k$ et par suite $a=0$ ou $b=0$. Si $b=0$, alors $p q \varepsilon=a^{2}$, donc $p q \varepsilon_{1}=a^{\prime 2}$ ou $p q=a^{\prime \prime 2}$, ce qui n'est pas possible car $\varepsilon_{1}$ est de norme -1 dans le premier cas et $\sqrt{p q} \notin k$ dans le deuxième cas. Si $a=0$ on aura $\varepsilon=-\varepsilon_{1} \sqrt{2} b^{2}$; en passant à la norme on trouve que \pm 2 est un carré dans $\mathbb{Q}$, ce qui est impossible. Donc la classe de $\mathcal{P} \mathcal{Q}$ est d'ordre 2, ainsi $C_{2, K}$ est engendré par les classes de $\mathcal{P}$ et $\mathcal{Q}$.

Montrons que $\mathcal{P} \mathcal{Q}$ capitule dans $K(\sqrt{p q})$. Comme dans le cas précédent, le problème est de chercher $\gamma$ dans $K(\sqrt{p q})$ tel que $\left(\gamma^{2}\right)=(p q)$ dans $K(\sqrt{p q})$; or ceci est vérifié en prenant $\gamma=\sqrt{p q}$. Ainsi $\mathcal{P} \mathcal{Q}$ capitule dans $F_{1}=K(\sqrt{p q})$.

En résumé, on a le théorème suivant :

THÉORÈmE 8. Soient $K=k\left(\sqrt{-p q \varepsilon_{1} \sqrt{2}}\right)$ où $\varepsilon_{1}$ est l'unité fondamentale de $k=\mathbb{Q}(\sqrt{2}), p, q$ deux nombres premiers différents $\equiv 1 \bmod 4$ tel que $\left(\frac{2}{p}\right)=\left(\frac{2}{q}\right)=-1, F_{1}=K(\sqrt{p q}), F_{2}=K(\sqrt{p}), F_{3}=K(\sqrt{q})$ et $K_{0}=$ $\mathbb{Q}(\sqrt{2}, \sqrt{p q})$. Donc :

(i) Si $Q_{K_{0}}=1$, alors les quatres classes de $C_{2, K}$ capitulent dans $F_{1}$ et dans chaque $F_{i}, i \in\{2,3\}$, deux classes seulement de $C_{2, K}$ capitulent.

(ii) Si $Q_{K_{0}}=2$, alors dans chaque extension $F_{i}, i \in\{1,2,3\}$, il existe exactement deux classes de $C_{2, K}$ qui capitulent.

Dans la suite on aura besoin du résultat suivant consernant le symbole du reste normique.

Proposition 7 (voir [9]). Soient $M$ un corps de nombres contenant les racines $m$-ièmes de l'unité, $L$ une extension finie de $M, \alpha \in M^{*}$ et $\beta \in L^{*}$. On note $P$ un idéal premier de $M$, et $\mathcal{P}$ un idéal premier de $L$ au-dessus de P. Alors

$$
\prod_{\mathcal{P}}\left(\frac{\beta, \alpha}{\mathcal{P}}\right)_{m}=\left(\frac{N_{L / M}(\beta), \alpha}{P}\right)_{m},
$$

où le produit est pris sur tous les premiers de $L$ qui sont au-dessus de $P$.

THÉORÈmE 9 . Soient $K=k\left(\sqrt{-p q \varepsilon_{1} \sqrt{2}}\right)$ où $\varepsilon_{1}$ est l'unité fondamentale de $k=\mathbb{Q}(\sqrt{2}), p$ et $q$ deux nombres premiers différents $\equiv 1 \bmod 4$ tels que $\left(\frac{2}{p}\right)=\left(\frac{2}{q}\right)=-1, K_{0}=\mathbb{Q}(\sqrt{2}, \sqrt{p q}), F_{1}=K(\sqrt{p q}), F_{2}=K(\sqrt{p})$, $F_{3}=K(\sqrt{q})$ et $G=\operatorname{Gal}\left(K_{2}^{(2)} / K\right)$. Alors $G$ est diédral ou quaternionique d'ordre $2^{m}(m \geq 3)$ suivant que $Q_{K_{0}}=1$ ou $Q_{K_{0}}=2$.

Démonstration. Si $Q_{K_{0}}=1$, alors les quatres classes de $C_{2, K}$ capitulent dans $F_{1}$ et dans chaque $F_{i}(i=2,3)$ deux classes seulement de $C_{2, K}$ capitulent, ainsi d'après le théorème $3, G$ est isomorphe à $D_{m}(m \geq 3)$. 
Si $Q_{K_{0}}=2$, alors dans chaque $F_{i}(i=1,2,3)$ deux classes seulement de $C_{2, K}$ capitulent. Soient $\mathcal{P}, \mathcal{Q}$ les idéaux premiers de $K$ au-dessus de $p$ et $q$ respectivement. Alors $\mathcal{P}$ capitule dans $F_{2}=K(\sqrt{p}), \mathcal{Q}$ capitule dans $F_{3}=K(\sqrt{q})$ et $\mathcal{P} \mathcal{Q}$ capitule dans $F_{1}=K(\sqrt{p q})$.

Montrons que $F_{1}$ est de type (A) et que $F_{2}$ et $F_{3}$ sont de type (B). En effet, pour $F_{2}$, soit $K^{\prime}=k\left(\sqrt{-q \varepsilon_{1} \sqrt{2}}\right)$; alors on a $K K^{\prime}=F_{2}, N_{K / k}(\mathcal{P})=p$ et $p$ est non ramifié dans $K^{\prime} / k$; ainsi pour montrer que $\mathcal{P}$ est inerte dans $F_{2} / K$, il suffit de montrer que $p$ est inerte dans $K^{\prime} / k$ (théorème de translation) et pour cela on calcule le symbole du reste normique $\left(\frac{p,-q \varepsilon_{1} \sqrt{2}}{p}\right)$. Puisque $p \in \mathbb{Q}$ est inerte dans $k / \mathbb{Q}$ et $-q \varepsilon_{1} \sqrt{2} \in k$, en utilisant la proposition 7 on trouve

$$
\left(\frac{p,-q \varepsilon_{1} \sqrt{2}}{p}\right)=\left(\frac{p, N_{k / \mathbb{Q}}\left(-q \varepsilon_{1} \sqrt{2}\right)}{p}\right)=\left(\frac{p, 2 q^{2}}{p}\right)=\left(\frac{2}{p}\right)=-1 ;
$$

ainsi $p$ est inerte dans $K^{\prime} / k$, d'où $\mathcal{P}$ est inerte dans $F_{2} / K$, ainsi $F_{2} / K$ est de type (B), et de même on montre que $\mathcal{Q}$ est inerte dans $F_{3} / K$, d'où $F_{3} / K$ est aussi de type (B).

Pour $F_{1}$, soit $K^{\prime}=k\left(\sqrt{-\varepsilon_{1} \sqrt{2}}\right)$; alors on a $K K^{\prime}=F_{1}, N_{K / k}(\mathcal{P})=p$ et $p$ est non ramifié dans $K^{\prime} / k$; ainsi pour montrer que $\mathcal{P}$ est inerte dans $F_{1} / K$, il suffit de montrer que $p$ est inerte dans $K^{\prime} / k$ (théorème de translation) et pour cela on calcule le symbole du reste normique $\left(\frac{p,-\varepsilon_{1} \sqrt{2}}{p}\right)$. En utilisant la proposition 7 , on trouve que $p \in \mathbb{Q}$ est inerte dans $k / \mathbb{Q}$ et $\varepsilon_{1} \sqrt{2} \in k$, ainsi

$$
\left(\frac{p,-\varepsilon_{1} \sqrt{2}}{p}\right)=\left(\frac{p, N_{k / \mathbb{Q}}\left(-\varepsilon_{1} \sqrt{2}\right)}{p}\right)=\left(\frac{p, 2}{p}\right)=\left(\frac{2}{p}\right)=-1 ;
$$

ainsi $p$ est inerte dans $K^{\prime} / k$, d'où $\mathcal{P}$ est inerte dans $F_{1} / K$, et de même on montre que $\mathcal{Q}$ est aussi inerte dans $F_{1} / K$, d'où $\mathcal{P} \mathcal{Q}$ est norme dans $F_{1} / K$, ainsi $F_{1} / K$ est de type (A), et en utilisant le théorème 3 , le groupe $G$ est isomorphe à $Q_{m}(m>3)$.

Remarque 2. Comme l'extension $F_{1} / K$ est de type $(A)$, d'après [13] le 2-groupe des classes de $F_{1}=K(\sqrt{p q})$ est cyclique.

THÉORÈME 10. Soient $K=k\left(\sqrt{-p q \varepsilon_{1} \sqrt{2}}\right)$ où $\varepsilon_{1}$ est l'unité fondamentale de $k=\mathbb{Q}(\sqrt{2})$, $p$ et $q$ deux nombres premiers différent $\equiv 1 \bmod 4$ tels que $\left(\frac{2}{p}\right)=\left(\frac{2}{q}\right)=-1$, et $F_{1}=K(\sqrt{p q})$. Alors $C_{2, F_{1}}$, la 2-partie $d u$ groupe de classes de $F_{1}$, est cyclique d'ordre $h_{2}\left(F_{1}\right)=2 Q_{K_{0}} h_{2}(p q)$ où $K_{0}=$ $\mathbb{Q}(\sqrt{2}, \sqrt{p q}), Q_{K_{0}}$ son indice des unités et $h_{2}(p q)$ est le 2 -nombre de classes de $\mathbb{Q}(\sqrt{p q})$.

Démonstration. Tout d'abord d'après la remarque $2, C_{2, F_{1}}$ est cyclique, et $F_{1} / k$ est une extension biquadratique normale de groupe de Galois de type $(2,2)$, de sous-extensions quadratiques $K, K^{\prime}=k\left(\sqrt{-\varepsilon_{1} \sqrt{2}}\right)$ et $K_{0}$; alors 
d'après la proposition 3 , on trouve que $h_{2}\left(F_{1}\right)=\frac{1}{2} q\left(F_{1}\right) h_{2}(K) h_{2}\left(K^{\prime}\right) h_{2}\left(K_{0}\right)$, car $d=2, \kappa=1, v=0$. Or $h_{2}(K)=4$, d'après [6] on a $h_{2}\left(K^{\prime}\right)=1$, d'après [18] on a $h_{2}\left(K_{0}\right)=\frac{1}{4} Q_{K_{0}} h_{2}(2) h_{2}(p q) h_{2}(2 p q)$, et comme $h_{2}(2)=1$ et $h_{2}(2 p q)=4$ (d'après [12]), on a $h_{2}\left(K_{0}\right)=Q_{K_{0}} h_{2}(p q)$; ainsi $h_{2}\left(F_{1}\right)=$ $2 q\left(F_{1}\right) Q_{K_{0}} h_{2}(p q)$. D'après le théorème $5, K_{0}$ et $F_{1}$ on même $\mathbf{S F U}$, donc $q\left(F_{1}\right)=1$, d'où le résultat.

REMARQUe 3 . Soient $K=k\left(\sqrt{-p q \varepsilon_{1} \sqrt{2}}\right)$ où $\varepsilon_{1}$ est l'unité fondamentale de $k=\mathbb{Q}(\sqrt{2}), p$ et $q$ deux nombres premiers différent $\equiv 1 \bmod 4$ tels que $\left(\frac{2}{p}\right)=\left(\frac{2}{q}\right)=-1$ et $G=\operatorname{Gal}\left(K_{2}^{(2)} / K\right)$. Alors $|G|=4 Q_{K_{0}} h_{2}(p q)$ où $K_{0}=$ $\mathbb{Q}(\sqrt{2}, \sqrt{p q}), Q_{K_{0}}$ est son indice des unités et $h_{2}(p q)$ est le 2-nombre des classes de $\mathbb{Q}(\sqrt{p q})$.

Démonstration. Soit $F_{1}=K(\sqrt{p q})$; alors $K_{2}^{(1)} / F_{1}$ est une extension non ramifiée et $C_{2, F_{1}}$ est cyclique, ainsi $F_{1}$ et $K_{2}^{(1)}$ ont même 2-corps des classes de Hilbert, à savoir $K_{2}^{(2)}$, donc $|G|=2 h_{2}\left(F_{1}\right)=4 Q_{K_{0}} h_{2}(p q)$.

Exemples numériques

(1) Soient

$$
\begin{aligned}
& K=k(\sqrt{-3.11(1+\sqrt{2}) \sqrt{2}}), \quad k=\mathbb{Q}(\sqrt{2}), \\
& F_{1}=K(\sqrt{3.11}), \quad F_{2}=K(\sqrt{-3}), \quad F_{3}=K(\sqrt{-11}) .
\end{aligned}
$$

Comme $3 \equiv 11 \equiv-1 \bmod 4$ et $\left(\frac{2}{3}\right)=\left(\frac{2}{11}\right)=-1$, d'après le théorème 7 on a $G \simeq \mathbb{Z} / 2 \mathbb{Z} \times \mathbb{Z} / 2 \mathbb{Z}$ et les quatre classes de $C_{2, K}$ capitulent dans chacune des extensions $F_{i} / K(i=1,2,3)$.

(2) Soient

$$
\begin{aligned}
& K=\mathbb{Q}(\sqrt{-3.19(2+\sqrt{2})}), \\
& F_{1}=K(\sqrt{3.19}), \quad F_{2}=K(\sqrt{-3}), \quad F_{3}=K(\sqrt{-19}) .
\end{aligned}
$$

Comme $3 \equiv 19 \equiv-1 \bmod 4$ et $\left(\frac{2}{3}\right)=\left(\frac{2}{19}\right)=-1$, d'après le théorème 7 on a $G \simeq \mathbb{Z} / 2 \mathbb{Z} \times \mathbb{Z} / 2 \mathbb{Z}$ et les quatre classes de $C_{2, K}$ capitulent dans chacune des extensions $F_{i} / K(i=1,2,3)$.

(3) Soient

$$
\begin{aligned}
& K=\mathbb{Q}(\sqrt{-67.83(2+\sqrt{2})}), \\
& F_{1}=K(\sqrt{67.83}), \quad F_{2}=K(\sqrt{-67}), \quad F_{3}=K(\sqrt{-83}) .
\end{aligned}
$$

Comme $67 \equiv 83 \equiv-1 \bmod 4$ et $\left(\frac{2}{67}\right)=\left(\frac{2}{83}\right)=-1$, d'après le théorème 7 le groupe $G$ est abélien et les quatre classes de $C_{2, K}$ capitulent dans chacune des extensions $F_{i} / K(i=1,2,3)$. 
(4) Soit $K=\mathbb{Q}(\sqrt{-p q(2+\sqrt{2})})$, avec $p, q$ deux nombres premiers tels que $p \equiv q \equiv 5 \bmod 8,\left(\frac{p}{q}\right)=1,\left(\frac{p}{q}\right)_{4}=-\left(\frac{q}{p}\right)_{4}$ et $K_{0}=\mathbb{Q}(\sqrt{2}, \sqrt{p q})$.

L'unité fondamentale de $\mathbb{Q}(\sqrt{p q})$ est de norme 1 , ainsi $Q_{K_{0}}=1$, par suite, en utilisant le théorème $9, G$ est diédral et les quatre classes de $C_{2, K}$ capitulent dans $F_{1}=K(\sqrt{p q})$, tandis que dans chacune des extensions $F_{2}=$ $K(\sqrt{p})$ et $F_{3}=K(\sqrt{q})$ deux classes seulement de $C_{2, K}$ capitulent. De plus, on sait que $h_{2}(p q)=2$, ainsi, en utilisant la remarque 3 , on trouve que $|G|=8$, ce qui donne que $G \simeq D_{3}$.

(5) Soient $K=\mathbb{Q}(\sqrt{-5.37(2+\sqrt{2})})$ et $K_{0}=\mathbb{Q}(\sqrt{2}, \sqrt{5.37})$; alors comme $Q_{K_{0}}=1$, donc, d'après le théorème $9, G$ est diédral et les quatre classes de $C_{2, K}$ capitulent dans $F_{1}=K(\sqrt{5.37})$, tandis que dans chacune des extensions $F_{2}=K(\sqrt{5})$ et $F_{3}=K(\sqrt{37})$ deux classes seulement de $C_{2, K}$ capitulent. De plus, comme $\left(\frac{5}{37}\right)=-1$, on a $h_{2}(5.37)=2$, ainsi, en utilisant la remarque 3 , on trouve que $|G|=8$, ce qui donne que $G \simeq D_{3}$.

(6) Soient $K=\mathbb{Q}(\sqrt{-5.29(2+\sqrt{2})})$ et $K_{0}=\mathbb{Q}(\sqrt{2}, \sqrt{5.29})$; alors comme $Q_{K_{0}}=1, G$ est diédral et les quatre classes de $C_{2, K}$ capitulent dans $F_{1}=$ $K(\sqrt{5.29})$, tandis que dans chacune des extensions $F_{2}=K(\sqrt{5})$ et $F_{3}=$ $K(\sqrt{29})$ deux classes seulement de $C_{2, K}$ capitulent, plus précisement comme $\left(\frac{5}{29}\right)_{4}=\left(\frac{29}{5}\right)_{4}=-1$, on a $h_{2}(5.29)=4$, ainsi $|G|=16$, ce qui donne que $G \simeq D_{4}$.

(7) Soient $K=\mathbb{Q}(\sqrt{-5.101(2+\sqrt{2})})$ et $K_{0}=\mathbb{Q}(\sqrt{2}, \sqrt{5.101})$; alors comme $Q_{K_{0}}=1, G$ est diédral et les quatre classes de $C_{2, K}$ capitulent dans $F_{1}=K(\sqrt{5.101})$, tandis que dans chacune des extensions $F_{2}=K(\sqrt{5})$ et $F_{3}=K(\sqrt{101})$ deux classes seulement de $C_{2, K}$ capitulent; en plus, comme $\left(\frac{5}{101}\right)_{4}=\left(\frac{101}{5}\right)_{4}=1$ et l'unité fondamentale de $\mathbb{Q}(\sqrt{5.101})$ est de norme 1 , on a $4 \mid h_{2}(5.101)$, ainsi 16||$G \mid$, ce qui donne que $G \simeq D_{m}$ où $m \geq 4$.

(8) Soient

$$
\begin{aligned}
& K=\mathbb{Q}(\sqrt{-5.13(2+\sqrt{2})}), \quad K_{0}=\mathbb{Q}(\sqrt{2}, \sqrt{5.13}), \\
& F_{1}=K(\sqrt{5.13}), \quad F_{2}=K(\sqrt{5}), \quad F_{3}=K(\sqrt{13}) .
\end{aligned}
$$

Comme $Q_{K_{0}}=2$, le groupe $G$ est quaternionique et dans chacune des extensions $F_{i}(i=1,2,3)$ deux classes seulement de $C_{2, K}$ capitulent, et comme $\left(\frac{5}{13}\right)=-1$, on a $h_{2}(5.13)=2$; ainsi, en utilisant la remarque 3 , on trouve que $|G|=16$, ce qui donne que $G \simeq Q_{4}$.

(9) Soient

$$
\begin{aligned}
& K=\mathbb{Q}(\sqrt{-53.61(2+\sqrt{2})}), \quad K_{0}=\mathbb{Q}(\sqrt{2}, \sqrt{53.61}), \\
& F_{1}=K(\sqrt{53.61}), \quad F_{2}=K(\sqrt{53}), \quad F_{3}=K(\sqrt{61}) .
\end{aligned}
$$

On a $Q_{K_{0}}=2$, ainsi $G$ est quaternionique et dans chacune des extensions 
$F_{i}(i=1,2,3)$ deux classes seulement de $C_{2, K}$ capitulent; en plus, comme $\left(\frac{53}{61}\right)=-1$, alors $h_{2}(53.61)=2$, ainsi, en utilisant la remarque 3 , on trouve que $|G|=16$, ce qui donne que $G \simeq Q_{4}$.

(10) Soient

$$
\begin{aligned}
& K=\mathbb{Q}(\sqrt{-13.101(2+\sqrt{2})}), \quad K_{0}=\mathbb{Q}(\sqrt{2}, \sqrt{13.101}), \\
& F_{1}=K(\sqrt{13.101}), \quad F_{2}=K(\sqrt{13}), \quad F_{3}=K(\sqrt{101}) .
\end{aligned}
$$

Alors, comme $Q_{K_{0}}=2$, donc en utilisant le théorème $9, G$ est quaternionique et dans chacune des extensions $F_{i}(i=1,2,3)$ deux classes seulement de $C_{2, K}$ capitulent, et comme $\left(\frac{13}{101}\right)_{4}=\left(\frac{101}{13}\right)_{4}=-1$, alors $h_{2}(13.101)=4$; ainsi $|G|=32$, ce qui donne que $G \simeq Q_{5}$.

(11) Soient

$$
\begin{aligned}
& K=\mathbb{Q}(\sqrt{-29.197(2+\sqrt{2})}), \quad K_{0}=\mathbb{Q}(\sqrt{2}, \sqrt{29.197}), \\
& F_{1}=K(\sqrt{29.197}), \quad F_{2}=K(\sqrt{29}), \quad F_{3}=K(\sqrt{197}) .
\end{aligned}
$$

Comme $Q_{K_{0}}=2$, d'après le théorème $9, G$ est quaternionique et dans chacune des extensions $F_{i}(i=1,2,3)$ deux classes seulement de $C_{2, K}$ capitulent, et puisque $\left(\frac{29}{197}\right)_{4}=\left(\frac{197}{29}\right)_{4}=1$ et l'unité fondamentale de $\mathbb{Q}(\sqrt{29.197})$ est de norme -1 , donc $8 \mid h_{2}(29.197)$, ainsi, en utilisant la remarque 3 , on a 64||$G \mid$, ce qui donne que $G \simeq Q_{m}$ où $m \geq 6$.

Remarque 4. Pour les résultats concernant le calcul du 2-nombre de classes des corps quadratiques sur $\mathbb{Q}$, voir P. Kaplan [12].

\section{Références}

[1] A. Azizi, Capitulation des 2-classes d'idéaux de $\mathbb{Q}(\sqrt{d}, i)$, thèse, Université Laval, Québec, 1993.

[2] - Unités de certains corps de nombres imaginaires et abéliens sur $\mathbb{Q}$, Ann. Sci. Math. Québec 23 (1999), 15-21.

[3] —, Sur la capitulation des 2-classes d'idéaux de $\mathbb{k}=\mathbb{Q}(\sqrt{2 p q}, i)$ où $p \equiv-q \equiv$ $1 \bmod 4$, Acta Arith. 94 (2000), 383-399.

[4] A. Azizi et A. Mouhib, Sur le rang du 2-groupe de classes de $\mathbb{Q}(\sqrt{m}, \sqrt{d})$ où $m=2$ ou un premier $p \equiv 1 \bmod 4$, Trans. Amer. Math. Soc. 353 (2001), 2741-2752.

[5] - - -, Capitulation des 2-classes d'idéaux de $\mathbb{Q}(\sqrt{2}, \sqrt{d})$ où d est un entier naturel sans facteurs carrés, Acta Arith. 109 (2003), 27-63.

[6] E. Brown and C. J. Parry, The 2-class group of certain biquadratic number fields I, J. Reine Angew. Math. 295 (1977), 61-71.

[7] -, -, The 2-class group of biquadratic number fields II, Pacific J. Math. 78 (1978), $11-26$.

[8] G. Gras, Sur les l-classes d'idéaux dans les extensions cycliques relatives de degré premier l, Ann. Inst. Fourier (Grenoble) 23 (1973), no. 3, 1-48, et no. 4, 1-44.

[9] H. Hasse, Neue Begründung der Theorie des Normenrestsymbols, J. Reine Angew. Math. 162 (1930), 134-143. 
[10] F. P. Heider und B. Schmithals, Zur Kapitulation der Idealklassen in unverzweigten primzyklischen Erweiterungen, ibid. 336 (1982), 1-25.

[11] M. Ishida, The Genus Fields of Algebraic Number Fields, Lecture Notes in Math. 555, Springer, 1976.

[12] P. Kaplan, Sur le 2-groupe des classes d'idéaux des corps quadratiques, J. Reine Angew. Math. 283/284 (1976), 313-363.

[13] H. Kisilevsky, Number fields with class number congruent to 4 mod 8 and Hilbert's Theorem 94, J. Number Theory 8 (1976), 271-279.

[14] T. Kubota, Über den bizyklischen biquadratischen Zahlkörper, Nagoya Math. J. 10 (1956), 65-85.

[15] S. Kuroda, Über den Dirichletschen Zahlkörper, J. Fac. Sci. Imp. Univ. Tokyo Sec. I 4 (1943), 383-406.

[16] F. Lemmermeyer, Ideal class groups of cyclotomic number fields I, Acta Arith. 72 (1995), 347-359.

[17] —, Kuroda's class number formula, ibid. 66 (1994), 245-260.

[18] H. Wada, On the class number and the unit group of certain algebraic number fields, Tokyo Univ. Fac. Sci. J. Ser. I 13 (1966), 201-209.

Département de Mathematiques

Faculté des Sciences

Université Mohammed 1

Oujda, Maroc

E-mail: abdelmalekazizi@yahoo.fr

talbimm@yahoo.fr 